\title{
Blood Products Component Preparation Facility
}

National Cancer Institute

\section{Source}

National Cancer Institute. Blood Products Component Preparation Facility. NCI

Thesaurus. Code C133302.

An intermediate processing facility that prepares components from blood collected by a mobile or fixed collection site but does not perform product testing. 